\title{
Continuous registration based on computed tomography for breathing motion compensation
}

\author{
Dominik Spinczyk ${ }^{1}$, Jaroslaw Zyłkowski², Tadeusz Wróblewski \\ 1Silesian University of Technology, Katowice, Poland \\ 2Second Department of Clinical Radiology, Medical University of Warsaw, Warsaw, Poland \\ ${ }^{3}$ Chair and Department of General, Transplant and Liver Surgery, Medical University of Warsaw, Warsaw, Poland
}

Videosurgery Miniinv 2013; 8 (4): 265-272

DOI: 10.5114/wiitm.2013.39505

\begin{abstract}
Introduction: Image guidance for intervention is applied for complex and difficult anatomical regions. Nowadays, it is typically used in neurosurgery, otolaryngology, orthopedics and dentistry. The application of the image-guided system for soft tissues is challenging due to various deformations caused by respiratory motion, tissue elasticity and peristalsis.

Aim: The main task for the presented approach is continuous registration of preoperative computed tomography (CT) and patient position in the operating room (OR) without touching the patient and compensation of breathing motion. This approach is being developed as a step to image-guided percutaneous liver RF tumor ablation.

Material and methods: Up to ten integrated radiological markers are placed on the patient's skin before CT scans. Then the anatomical model based on CT images is calculated. Point-to-point registration based on the Horn algorithm during a few breathing cycles is performed using a videometric tracking system. The transformation which corresponds to the minimum fiducial registration error (FRE) is found during the registration and it is treated as the initial transformation for calculating local deformation field of breathing motion compensation based on the spline approach.

Results: For manual registration of the abdominal phantom, the mean values of target registration error (TRE), fiducial localization error (FLE) and FRE are all below $4 \mathrm{~mm}$ for the rigid transformation and are below $1 \mathrm{~mm}$ for the affine transformation. For the patient's data they are all below $9 \mathrm{~mm}$ and $6 \mathrm{~mm}$, respectively. For the automatic method, different marker configurations have been evaluated while dividing the respiratory cycle into inhale and exhale. Average median values for FRE, TRE rigid estimation and TRE based on spline deformation were $15.56 \mathrm{~mm}, 0.82 \mathrm{~mm}$ and $7.21 \mathrm{~mm}$ respectively.

Conclusions: In this application two registration methods of abdominal preoperative CT anatomical model and physical patient position in OR were presented and compared. The presented approach is being developed as a step to image-guided percutaneous liver radiofrequency ablation tumor ablation. Implementation of the automated registration method to clinical practice is easier because of shortening of preparation time in $O R$, no necessity of touching the patient, and no dependency on the physician's experience.
\end{abstract}

Key words: percutaneous ablation, preoperative anatomical model registration, respiratory motion compensation, image-guided intervention. 


\section{Introduction}

Image guidance for intervention is applied for complex and difficult anatomical regions. Nowadays, it is typically used in neurosurgery, otolaryngology, orthopedics and dentistry [1-4]. The application of the image-guided system for soft tissues is challenging due to various deformations caused by respiratory motion, tissue elasticity and peristalsis $[5,6]$.

Most patients with liver tumors are not candidates for surgical resections, because of unfavorable pathological sites of tumor, widespread metastases and poor general physical condition. For these patients there is still a chance in minimally invasive, percutaneous ablations of lesions. The major task of these procedures is accurate needle placement. The conventional method for guiding needle insertion is ultrasound imaging, but small and deeply situated lesions are difficult to identify. The main task for the presented approach is continuous registration of preoperative computed tomography (CT) and patient position in the operating room (OR) without touching the patient and compensation of breathing motion.

Nowadays, this kind of system is at the development stage [7]. One application of the Image-Guided Surgery Toolkit [8] is CT-based liver tumor biopsy [9]. Rigid transformation and the Horn algorithm [10] are used to register the preoperative model and the patient's position. The needle is tracked by an optical tracking system. Fiducial markers, entry and target points are manually selected in $\mathrm{CT}$ images. Target registration error based on Fitzpatrick estimation [11] is calculated. Banovac et al. [12] use an electromagnetic tracking system to traverse needles into the radiopaque tumor target as well as point-to-point registration algorithm. The average registration error between the CT coordinate system and electromagnetic coordinate system was $1.4 \pm 0.3 \mathrm{~mm}$ ) in the phantom and $1.9 \pm 0.3 \mathrm{~mm}$ ) in the swine. Maier-Hein et al. [13, 14] proposed a CTbased, optical tracking system and a real-time deformation model to continuously estimate the position of the target in the liver. A spline-based approach was used to capture local deformations between the preoperative CT anatomical model and the patient's position in the OR. The proposed approach was tested combining internal and external markers in a customdesigned respiratory liver motion simulator and in swine tissues. Diagonal arrangement of the needleshaped fiducials yielded the best results. The rest of the article presents the proposed registration method, which is used to compensate respiratory movements. The proposed method consists of two steps: rigid and non-rigid registration.

\section{Aim}

The main task for the presented approach is continuous registration of preoperative $\mathrm{CT}$ and patient position in the OR without touching the patient and compensation of breathing motion. This approach is being developed as a step to image-guided percutaneous liver radiofrequency ablation (RF) tumor ablation.

\section{Material and methods}

Registering a preoperative anatomical model and the patient's position starts with a rigid registration. In our approach, the initial registration is based on radiological markers. The presented method uses Beekley CT Spots skin markers which are attached to the patient's abdominal skin before the CT examination and then are used for patient registration. The selected markers were used because they do not produce artifacts on CT images and it is possible to pinpoint their position. Markers are manually selected: in the CT abdominal examination and on the patient's skin. At the very beginning (it was a pilot study) the Polaris Vicra tracking system was used and markers on the patient's skin were manually selected. We used up to ten markers to test various configurations of the marker's positions relative to the target point. West et al. [9] demonstrated the importance of configuration of markers' positions relative to the target point to the registration error. The description of anatomical position of the skin markers is presented in Figure 1 and Table I. The following configuration of markers against the central marker were tested: all, up-down, left-right and diagonal. For each configuration, the central marker was set as a target. To find rigid mapping between two Cartesian coordinate systems, data must include three or more corresponding non-collinear points. Horn [10] proposed a closed form solution based on a least-squares formulation. Optimal rotation and translation matrices are found using singular value decomposition (SVD) of a correlation matrix:

$$
\operatorname{SVD}(C)=\operatorname{Udiag}\left(\sigma_{i}\right) V
$$

where $C=\sum_{i=1}^{n} S_{i}{ }^{T} T_{i}$ is the correlation matrix, Si are points in the first Cartesian coordinate system, Ti are 
A

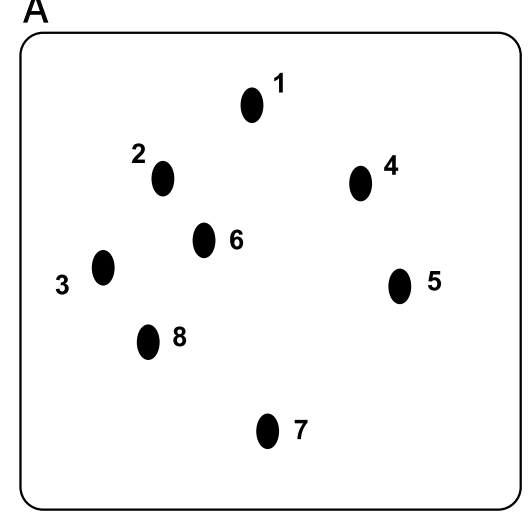

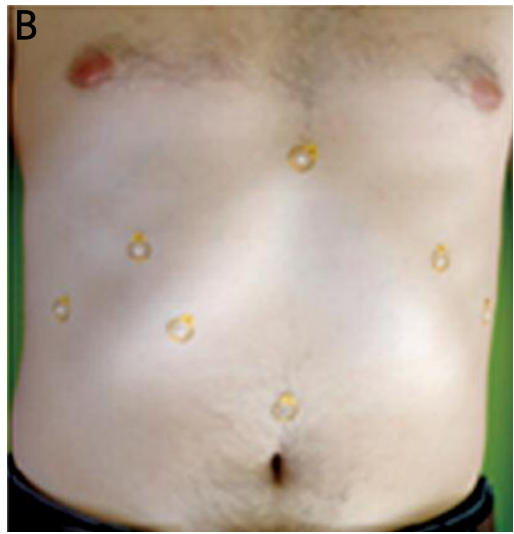

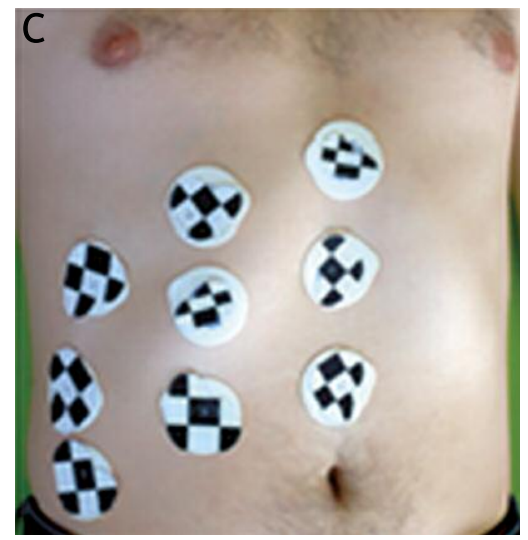

Figure $1 \mathrm{~A}-\mathrm{C}$. Diagram of patient's abdominal marker positions

Table I. Anatomical position of skin markers for manual registration

\begin{tabular}{|lll|}
\hline $\begin{array}{l}\text { Marker } \\
\text { number }\end{array}$ & Anatomical location & Grounds \\
\hline 1 & Xiphoid process & Point fixed to bony structures in the midline of the body \\
\hline 2 & Intersection of midclavicular line and right costal margin & Anatomical point fixed to bony structures \\
\hline 3 & Intersection of anterior axillary line and right costal margin & Anatomical point fixed to bony structures \\
\hline 4 & Intersection of midclavicular line and left costal margin & Anatomical point fixed to bony structures \\
\hline 5 & Intersection of anterior axillary line and left costal margin & Anatomical point fixed to bony structures \\
\hline 6 & First control point on the abdominal surface & Registration accuracy validation \\
\hline 7 & Umbilicus & Anatomical point not fixed to bony structures \\
\hline 8 & Second control point on the abdominal surface & Registration accuracy validation \\
\hline
\end{tabular}

points in the second Cartesian coordinate system, $\sigma_{i}$ are non-negative singular values of the correlation matrix and $U$ and $V$ are orthonormal matrices,

$R o t=U\left[\begin{array}{lll}1 & 0 & 0 \\ 0 & 1 & 0 \\ 0 & 0 & \operatorname{det}\left(U V^{\top}\right)\end{array}\right] V^{T}$

and

Trans $=\bar{T}-\operatorname{Rot}(\bar{S})$

where $\bar{S}, \bar{T}$ are average values of the point coordinates in the first and second coordinate systems, respectively.

The method has been evaluated on imaging data and patients with any liver diseases from the Second Department of Clinical Radiology for patients on the Chair and Department of General, Transplant and Liver
Surgery at the Medical University of Warsaw after acceptance of the ethics committee of the Medical University of Warsaw (GE Light Speed 16 Pro CT Scanner; Contrast medium: Iomeron 400 or Ultravist 370; Intravenous bolus: $10 \mathrm{ml}$ of $0.9 \% \mathrm{NaCl}, 1 \mathrm{ml} / \mathrm{kg}$ contrast medium and thereafter $30 \mathrm{ml}$ of $0.9 \% \mathrm{NaCl}$ all with $6 \mathrm{ml} / \mathrm{s}$ infusion speed). In order to assess the results, FLE, FRE and TRE were used. Following the Fitzpatrick estimation of registration error [10], different markers were evaluated as targets. The results of this study are presented in the next section. After the pilot study we found a few disadvantages of the manual registration: the necessity of touching the patient's skin manually, prolonging the time of the procedure, and dependence of accuracy on the physician's experience.

Due to the disadvantages we decided to implement an automatic continuous registration. The automated method in this case means no need for multi- 
ple manual indication of the position of markers on the patient. Position of markers is determined not once, but several times during the respiratory cycle. We used the videometric tracking system of Micron Tracker Claron Hx. 40. Claron Hx 40 is stereo camera which tracks a unique black-white template with an error of less than $0.5 \mathrm{~mm}$ after calibration. We put the markers on the patient before CT scans but unlike in a manual registration all markers were placed on one (right) side of the abdomen for visibility of the tracking system. We constantly grab the position of the marker (a few times a second) and calculate the FRE error between the DICOM coordinate system and patient (tracker) coordinate system. The resulting transform is organized by the error FRE and the transform which corresponds to the minimum is treated as the result of rigid registration.

\section{Breathing motion compensation}

Breathing motion compensation is necessary to select a proper breathing phase for needle insertion and estimate the target movement.

For breathing motion compensation, a local deformation field based on displacement of tracking skin markers is calculated. The set of marker positions (in the tracker coordinate system) during tracking time is treated as reference points for the local deformation field, based on a spline approach [15, 16]. The deformation equation:

$$
F(x)=D^{T} \times G(x)+A x+b
$$

calculates a new position of point $\mathrm{x}$ in space, based on spline coefficients matrix $D$ and affine transformation $A x+b$. Based on the position of tracking skin markers in the specific time and after a time interval, unknown spline coefficients of the current deformation field are found and then this field can be used to estimate the new target position. To evaluate the correctness of deformation one marker is treated as a target. Due to TRE estimation [11] usually the central marker is treated as a target. The transformation obtained for minimum FRE in the rigid registration step is used to translate the target from the DICOM coordinate system to the tracker coordinate system.

\section{Selecting proper breathing phase for needle insertion}

For tracking breathing, the marker positions are continuously measured by the videometric tracking system. Time course of marker positions is analyzed. Breathing cycles are detected based on local extremes. The breath is the process variable over time, so there is a need for averaging it $[17,18]$. Amplitude of respiratory motion is not measured directly, but through the respiratory phase. The filtering signal derived from the so-called breathing bag with a Gaussian filter to unambiguously determine the minimum and maximum is applied. A mean of the maximum breathing phase is assigned by the formula (assuming zero breathing phase for minima) [18]:

$$
\bar{\emptyset}^{\max }=\frac{1}{N^{\max }} \sum_{i=1}^{N^{\max }} \frac{t_{i}^{\max }-t_{i}^{\min }}{t_{i+1}^{\min }-t_{i}^{\min }}
$$

where $N^{\max }$ is the number of the observed breathing cycles and $t_{i}^{\max }$ and $t_{i}^{\min }$ are moments of time corresponding to the next extremes of marker position, and respiratory phase, for any time $t j$, is interpolated linearly [18]:

$$
\begin{aligned}
& \left(\frac{t_{j}-t_{i}^{\min }}{t_{i}^{\max }-t_{i}^{\min }}\right) \bar{\Phi}^{\max } ; \quad \text { for } t_{i}^{\min } \leq t_{j}<t_{i}^{\max } ; \\
& \bar{\emptyset}^{\max }+\left(\frac{t_{j}-t_{i}^{\max }}{t_{i+1}^{\min }-t_{i}^{\max }}\right)\left(1-\bar{\emptyset}^{\max }\right) ; \text { for } t_{i}^{\max } \leq t_{j}<t_{i+1}^{\min }
\end{aligned}
$$

Calculating the breathing phase allows breathing data to be grouped from many breathing cycles and also data to be compared between patients.

\section{Results \\ Manual rigid registration}

The rigid registration results of a preoperative CT anatomical model and a physical patient position are presented both for the phantom (Table II) and patient data (Table III). To check the dependence of the results on the operator and at the same time not to extend too much time with the patient, the method was evaluated by two operators.

For the manual registration of the abdominal phantom, the mean values of target registration error TRE, FLE, FRE are all below $4 \mathrm{~mm}$ for the rigid transformation and are below $1 \mathrm{~mm}$ for the affine transformation. For the patient's data they are all below $9 \mathrm{~mm}$ and $6 \mathrm{~mm}$, respectively. The smallest errors are obtained for the target position located in the center 
Table II. Errors of phantom markers registration using the rigid or affine transformation in millimeters (Polaris Vicra)

\begin{tabular}{|lcccccccc|}
\hline \multirow{2}{*}{\begin{tabular}{l} 
Eind of error \\
\cline { 2 - 9 }
\end{tabular}} & \multicolumn{9}{c}{ Rigid registration } & \multicolumn{3}{c|}{ Apror value } \\
\cline { 2 - 10 } & \multicolumn{2}{c}{ Operator 1 } & \multicolumn{2}{c}{ Operator 2 } & \multicolumn{2}{c|}{ Operator 1 } & Operator 2 \\
\cline { 2 - 10 } & Case 1 & Case 2 & Case 1 & Case 2 & Case 1 & Case 2 & Case 1 & Case 2 \\
\hline FRE & 2.08 & 1.91 & 1.56 & 4.33 & 0.52 & 0.52 & 0.87 & 1.04 \\
\hline TRE marker 1 & 3.29 & 2.77 & 2.60 & 7.27 & 0.52 & 0.69 & 0.87 & 1.04 \\
\hline TRE marker 5 & 1.56 & 1.21 & 1.04 & 2.94 & 0.35 & 0.52 & 0.35 & 0.52 \\
\hline TRE marker 9 & 1.73 & 2.77 & 2.25 & 6.58 & 0.52 & 0.52 & 0.87 & 1.04 \\
\hline TRE marker 3 & 2.42 & 2.77 & 1.91 & 5.37 & 0.52 & 0.52 & 0.87 & 0.87 \\
\hline TRE marker 8 & 4.50 & 3.81 & 3.12 & 7.62 & 0.69 & 0.69 & 1.04 & 1.21 \\
\hline FLE & 2.60 & 2.42 & 2.08 & 5.54 & 0.35 & 0.52 & 0.69 & 0.69 \\
\hline
\end{tabular}

Table III. Errors of patient's markers registration using the rigid or affine transformation for markers configuration: 8-4-5-2-6 in millimeters (using Polaris Vicra)

\begin{tabular}{|lcccc|}
\hline \multirow{2}{*}{\begin{tabular}{l} 
Kind of error \\
\cline { 2 - 5 }
\end{tabular}} & \multicolumn{2}{c}{ Rigid registration } & \multicolumn{2}{c|}{ Affine registration } \\
\cline { 2 - 5 } & Operator 1 & Operator 2 & Operator 1 & Operator 2 \\
\hline FRE & 7.1 & 5.0 & 4.7 & 3.1 \\
\hline TRE marker 1 & 11.4 & 7.8 & 7.4 & 3.8 \\
\hline TRE marker 5 & 7.3 & 5.0 & 4.7 & 5.0 \\
\hline TRE marker 9 & 11.8 & 8.1 & 7.6 & 4.0 \\
\hline TRE marker 3 & 9.0 & 6.6 & 5.7 & 2.3 \\
\hline TRE marker 8 & 5.0 & 3.5 & 3.3 & 4.0 \\
\hline FLE & 9.2 & 6.6 & 5.9 & 3.9 \\
\hline
\end{tabular}

of the marker coordinate system (target 5 in Figure 2 - Table II and target 6 in Figure 2 - Table III).

\section{Automatic continuous rigid registration and respiratory motion compensation}

The results of automatic registration of the preoperative CT anatomical model and a physical patient position are presented below. The anatomical location of markers is presented in Table III. Due to the field of view for the Micron Tracking system we restricted the area of marker placement to one side of the patient's body. The estimation of a new target position is calculated following the deformation field equation. Table IV presents two cases for a breathing phase divided into three groups:

- exhale $(0.85 \leq$ breathing_phase $\leq 0.15)$,

- middle $(0.15<$ breathing_phase $\leq 0.3$ or $0.7 \leq$ breathing_phase $<0.85)$,

- inhale $(0.3<$ breathing_phase $<0.7)$.

\section{Discussion}

There are some patients who demonstrate a little difference in rigid registration error in the whole breathing cycle. The error of target position estima- 
A

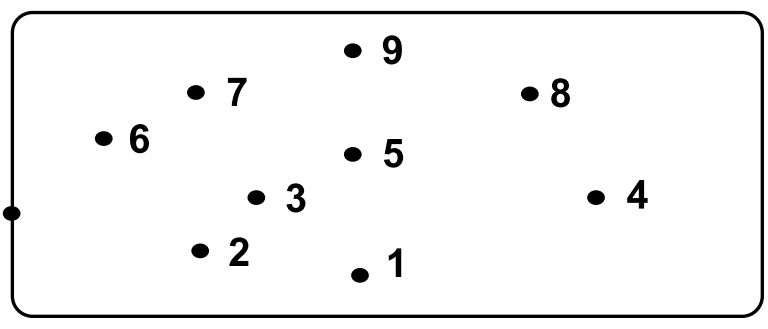

Figure 2 A, B. Diagram of phantom abdominal marker positions

Table IV. Errors of patient's markers registration for three breathing phase groups

\begin{tabular}{|llcc|}
\hline Breathing phase & Error type & \multicolumn{2}{c|}{ Median value } \\
\cline { 2 - 4 } & & Case 3 & Case 4 \\
\hline \multirow{3}{*}{ Exhale } & FRE_rigid & 7.04 & 4.26 \\
\cline { 2 - 4 } & TRE_spline & 4.36 & 1.08 \\
\cline { 2 - 4 } & TRE_rigid & 0.57 & 0.80 \\
\hline Middle & FRE_rigid & 7.05 & 7.05 \\
\cline { 2 - 4 } & TRE_spline & 4.37 & 1.57 \\
\cline { 2 - 4 } & TRE_rigid & 1.39 & 0.49 \\
\hline \multirow{3}{*}{ Inhale } & FRE_rigid & 7.1 & 7.73 \\
\cline { 2 - 4 } & TRE_spline & 4.44 & 1.91 \\
\cline { 2 - 4 } & TRE_rigid & 1.8 & 1.09 \\
\hline
\end{tabular}

Table V. Errors of patient's markers registration for median FRE

\begin{tabular}{|llcc|}
\hline \multirow{2}{*}{$\begin{array}{l}\text { Median FRE } \\
\text { breathing phase }\end{array}$} & Error type & \multicolumn{2}{c|}{ Median value } \\
\cline { 2 - 4 } & & Case 3 & Case 4 \\
\hline Exhale & FRE_rigid & 6.81 & 2.44 \\
\cline { 2 - 4 } & TRE_spline & 4.54 & 1.18 \\
\cline { 2 - 4 } & TRE_rigid & 1.41 & 0.80 \\
\hline Inhale & FRE_rigid & 7.18 & 9.76 \\
\cline { 2 - 4 } & TRE_spline & 4.16 & 2.63 \\
\cline { 2 - 4 } & TRE_rigid & 1.23 & 0.85 \\
\hline
\end{tabular}

tion for a specific patient and for rigid and spline estimations is similar for the whole breathing cycle, but generally it rises with an increase in respiratory

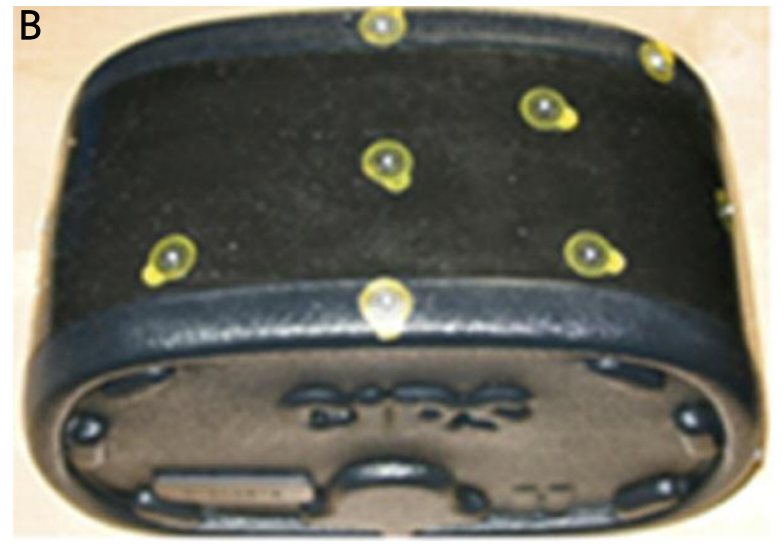

phase difference between preoperative data and a temporary breathing phase. Median of rigid fiducial registration error was used as a criterion to distinguish an inhale and exhale phase $[13,14]$. Table $V$ presents results based on this criterion (for the same patient as in Table IV).

Table VI presents registration and target position estimation errors for different marker configurations. Reconfiguring the markers will change registration errors.

Meier et al. $[13,14]$ report worse performance of target position estimation for left-right marker configuration for studies in swine. The results obtained generally do not support this rule. Due to big differences between patients (Table VII) it is necessary to treat each patient individually. One possible solution is the initial use of more markers (e.g. 8 to 10) during $C T$ examination and then after nodule localization in the CT preoperative anatomical model it is possible to test different configurations of markers before intervention to select the best one. The final number of proposed markers is limited by the line of sight between markers and the camera, which is distorted by an ultrasound head and ablation needle.

Implementation of the automated registration method to clinical practice is easier because of shortening of preparation time in $\mathrm{OR}$, no necessity of touching the patient, and no dependency on the physician's experience. In OR liver tumor ablation is usually performed in general anesthesia, so breathing is more regular than free breathing. As regards the image-guided system for percutaneous liver RF tumor ablation, the problem is to synchronize a respiratory phase for which the static preoperative CT anatomical model was generated. Using a shape con- 
serving mattress, the observation of marker movement and real time FRE calculation helps to select the best respiratory phase for needle insertion.

In this application two registration methods of abdominal preoperative $\mathrm{CT}$ and physical patient position in OR were presented and compared. This approach is being developed as a step to imageguided percutaneous liver RF tumor ablation. The proposed equation of deformation is calculated in real time. Assuming regular breathing conducted by the respirator, recurrence of patient position and short time between CT study and procedure, the proposed solution could be used to synchronize the respiratory phase for which the static preoperative CT anatomical model was generated, which is one of the most challenging steps in RF ablation.

In the absence of a direct correlation between the movements of the abdominal area and movement of internal organs to get more reliable registration results in the next step we want to apply 4D magnetic resonance or computed tomography imaging and build an internal organ motion model to correlate internal and external breathing motion.

\section{Acknowledgments}

The authors would like to acknowledge financial support from the Polish National Centre for Research and Development (NCBiR), grant no. LIDER/ 03/47/L-1/NCBiR/2010 "Development of planning system and computer aided minimally invasive surgery for hepatic and metastatic liver carcinoma localization, diagnosis and destruction". The method has been evaluated on imaging data and patients with any liver diseases from the Second Department of Clinical Radiology for patients on the Chair and Department of General, Transplant and Liver Surgery at the Medical University of War-
Table Vl. Errors of patient's markers registration for different marker configuration

\begin{tabular}{|c|c|c|c|c|}
\hline \multirow{2}{*}{$\begin{array}{l}\text { Markers } \\
\text { configu- } \\
\text { ration }\end{array}$} & \multirow{2}{*}{$\begin{array}{c}\text { Median FRE } \\
\text { breathing } \\
\text { phase }\end{array}$} & \multirow[t]{2}{*}{ Error type } & \multicolumn{2}{|c|}{ Median value } \\
\hline & & & Case 3 & Case 4 \\
\hline \multirow[t]{6}{*}{ 2_updown } & \multirow[t]{3}{*}{ Exhale } & FRE_rigid & 8.16 & 6.67 \\
\hline & & TRE_spline & 2.28 & 1.17 \\
\hline & & TRE_rigid & 0.12 & 0.74 \\
\hline & \multirow[t]{3}{*}{ Inhale } & FRE_rigid & 9.96 & 9.82 \\
\hline & & TRE_spline & 3.18 & 0.94 \\
\hline & & TRE_rigid & 3.32 & 0.65 \\
\hline \multirow[t]{6}{*}{ 2_diagonal } & \multirow[t]{3}{*}{ Exhale } & FRE_rigid & 6.58 & 1.66 \\
\hline & & TRE_spline & 4.54 & 0.80 \\
\hline & & TRE_rigid & 1.96 & 1.30 \\
\hline & \multirow[t]{3}{*}{ Inhale } & FRE_rigid & 7.13 & 1.86 \\
\hline & & TRE_spline & 2.95 & 0.94 \\
\hline & & TRE_rigid & 1.44 & 1.94 \\
\hline \multirow[t]{6}{*}{ 2_leftright } & \multirow[t]{3}{*}{ Exhale } & FRE_rigid & 4.79 & 4.07 \\
\hline & & TRE_spline & 0.85 & 1.36 \\
\hline & & TRE_rigid & 0.6 & 0.86 \\
\hline & \multirow[t]{3}{*}{ Inhale } & FRE_rigid & 4.93 & 4.21 \\
\hline & & TRE_spline & 0.71 & 1.34 \\
\hline & & TRE_rigid & 0.68 & 0.94 \\
\hline \multirow[t]{6}{*}{ All } & \multirow[t]{3}{*}{ Exhale } & FRE_rigid & 7.09 & 8.58 \\
\hline & & TRE_spline & 4.47 & 10.24 \\
\hline & & TRE_rigid & 0.47 & 1.04 \\
\hline & \multirow[t]{3}{*}{ Inhale } & FRE_rigid & 7.18 & 9.78 \\
\hline & & TRE_spline & 4.58 & 9.07 \\
\hline & & TRE_rigid & 1.08 & 1.14 \\
\hline
\end{tabular}

Table VII. Errors of patient's markers registration for median FRE

\begin{tabular}{|c|c|c|c|c|c|c|c|c|c|}
\hline \multirow[t]{3}{*}{ Error type } & \multicolumn{9}{|c|}{ Median value } \\
\hline & \multicolumn{3}{|c|}{ Minimum } & \multicolumn{3}{|c|}{ Maximum } & \multicolumn{3}{|c|}{ Mean } \\
\hline & Exhale & Inhale & Total & Exhale & Inhale & Total & Exhale & Inhale & Total \\
\hline FRE_rigid & 2.27 & 3.09 & 3.07 & 32.8 & 52 & 51.69 & 9.37 & 16.75 & 15.56 \\
\hline TRE_spline & 1.07 & 1.42 & 1.39 & 6.92 & 112.88 & 52 & 2.97 & 12.82 & 7.21 \\
\hline TRE_rigid & 0.34 & 0.3 & 0.29 & 1.78 & 1.23 & 1.69 & 0.85 & 2.43 & 0.82 \\
\hline
\end{tabular}


saw after acceptance of the ethics committee of the Medical University of Warsaw.

\section{References}

1. Strauss G, Koulechov K, Bahner J, et al. Evaluation of navigation system for ENT with surgical efficiency criteria. Laryngoscope 2006; 116: 564-72.

2. Woodworth BA, Chiu AG, Cohen NA, et al. Real-time computed tomography image update for endoscopic skull base surgery. J Laryngol Otol 2008; 122: 361-5.

3. Weber S, Lueth TC. Navigation of bone alignment osteotomies of the tibia. Int J Med Robot Comput Assist Surg 2008, 1: 98-107.

4. Shinoura N, Tabei Y, Yamada R, et al. Continuous intrathecal treatment with methotrexate via subcutaneous port: implication for leptomeningeal dissemination of malignant tumors. J Neurooncol 2008; 87: 309-16.

5. von Siebenthal M, Szekely G. 4D Imaging of respiratory organ motion and its variability. Phys Med Biol 2007; 52: 1547-64.

6. von Siebenthal M, Szekely G, Lomax A. Inter-subject modelling of liver deformation during radiation therapy. Med Image Comput Comput Assist Interv 2007; 10: 659-66.

7. Soo J, Kai Y. Interventional navigation systems for treatment of unresectable liver tumor. Med Biol Eng Comput 2010; 48: 103-11.

8. Cleary K, Enquobahrie A, Yaniv Z, et al. The image-guided surgery toolkit IGSTK: an open source C++ software toolkit. J Digit Imaging 2007; 20 Suppl. 1: 21-33.

9. West J, Fitzpatrick J, Toms S, et al. Fiducial point placement and the accuracy of point-based, rigid body registration. Neurosurgery 2001; 48: 810-6.

10. Horn B. Closed form solution of absolute orientation using unit quaterions. J Opt Soc Am A 1987; 4: 629-42.

11. Fitzpatrick JM, West JB, Mauer CR Jr. Predicting error in rigid-body point-based registration. IEEE Trans Med Imaging 2008; 17: 694-702.

12. Banovac F, Tang J, Xu S, et al. Precision targeting of liver lesions using a novel electromagnetic navigation device in physiologic phantom and swine. Med Phys 2005; 32: 2698-705.

13. Maier-Hein L, Tekbas A, Franz A, et al. On combining internal and external fiducials for liver motion compensation. Computer Aided Surgery 2008; 13: 369-76.

14. Maier-Hein L, Muller SA, Pianka F, et al. Respiratory motion compensation for CT-guided interventions in the liver. Comput Aided Surg 2008; 13: 125-38.

15. Davis H, Khotanzad A, Flaming D, Harms S. A physics-based coordinate transformation for 3-D Image Matching. IEEE Transactions on Medical Imaging 1997; 16: 317-28.

16. McClelland JR, Blackall JM, Tarte S, et al. A continuous 4D motion model from multiple respiratory cycles for use in lung radiotherapy. Medical Physics 2006; 33: 3348-58.

17. Siebenthal von M, Szekely G, Lomax AJ, Cattin PC. Systematic errors in respiratory gating due to intrafraction deformations of the liver. Medical Physics 2007; 34: 3620-9.

18. Rijkhorst E, Heanes D, Odille F, et al. Simulating dynamic ultrasound using MR-derived motion models to assess respiratory synchronisation for image-guided liver interventions. Lecture Notes in Computer Science 2010; 6135: 113-23.

Received: 22.07.2013, accepted: 24.11.2013. 